Voix et Images

voixetimages

\title{
Témoignage : La leçon des choses
}

\section{Jean-Claude Brochu}

Volume 12, numéro 2 (35), hiver 1987

Jacques Brault

URI : https://id.erudit.org/iderudit/200630ar

DOI : https://doi.org/10.7202/200630ar

Aller au sommaire du numéro

Éditeur(s)

Université du Québec à Montréal

ISSN

0318-9201 (imprimé)

1705-933X (numérique)

Découvrir la revue

Citer ce document

Brochu, J.-C. (1987). Témoignage : La leçon des choses. Voix et Images, 12(2),

250-254. https://doi.org/10.7202/200630ar

Ce document est protégé par la loi sur le droit d'auteur. L'utilisation des services d'Érudit (y compris la reproduction) est assujettie à sa politique d'utilisation que vous pouvez consulter en ligne.

https://apropos.erudit.org/fr/usagers/politique-dutilisation/
Cet article est diffusé et préservé par Érudit.

Érudit est un consortium interuniversitaire sans but lucratif composé de l’Université de Montréal, l'Université Laval et l'Université du Québec à Montréal. Il a pour mission la promotion et la valorisation de la recherche. https://www.erudit.org/fr/ 


\title{
Témoignage: \\ La leçon des choses
}

\author{
par Jean-Claude Brochu, Université de Montréal
}

Ainsi tourne la parole autour du livre: lire. écrire: d'un désir d̀ l'autre va toute littérature

(Roland Barthes, Critique et vérité, p. 79).

À Rimouski, dans une université - où nous voulions, là aussi, «bien mériter de la littérature" - et oì la Poésie ce matin figurait au programme d'un cours sur la poésie québécoise contemporaine, nous nous demandions naïvement pourquoi le poète avait résisté à la tentation d'une forme plus près de la prose avant les «enluminures». Dans la première. la troisième et la cinquième. l'auteur rétablit la ponctuation. fait des paragraphes plutôt que des strophes et déploie son texte apparemment plus sur des lignes que sur des vers... Deux années plus tard, alors que je posais la question à Jacques Brault au cours de notre première rencontre, il me dit que cela relevait de mon travail, que c'était affaire de lecture. C'était la première fois qu'il m'enseignait quelque chose et ce fut mon premier devoir. Je l'accomplis pour moi seul et cherchai la réponse "du côté du silence». Peut-être que le désir. dont il est question dans ces poèmes, s'accommode moins des silences ostensibles -blancs autour et dans le texte - et qu'il s'adapte mieux aux silences convenus - «émietté/s/» - de la ponctuation, du souffle? En amenuisant le silence autour du désir, le poète ne nous désigne-t-il pas tout le silence dans le désir même qui remonte à la rumeur du "commencement de notre âge"?

Ces contacts avec le poète et l'homme constituent en quelque sorte une leçon pré-inaugurale; depuis, $j^{\prime}$ ai suivi tous ses cours ${ }^{1}$. Deux ans de présence devant ce maître qui réalisait chaque semaine une promesse - une espérance - celle de faire naître quelque chose. Jacques Brault n’a jamais déçu; il n'avait qu'à être. C'est pourquoi il ne faudra pas se méprendre: même s'il y a bien deux ou trois saillies au début du texte et une échappée sur l'argumentation didactique de Jacques Brault, je souhaiterais offrir une vision tout en intériorité du professeur dont la philosophie (de la littérature) enveloppait les cours comme un climat nécessaire. Je pense que c'est toujours cela qui subsiste malgré nous: l'esprit, ou le sens profond et unificateur, plus que la lettre. Je me souviens, par exemple, que Jacques Brault opposait à l'indécision un parti pris de justesse basée sur l'observation et qu'il dégageait pour nous la leçon des choses un peu à la façon de Colette en qui il voyait le meilleur appareil sensitif de la littérature, c'est-à-dire en dénonçant les aberrations comme la blancheur de la neige et en nous initiant à la vraie couleur de l'aube ou à la lumière de Corot. Les pages qui suivent sont donc portées par une idée de la chose littéraire relancée tantôt par l'écriture, tantôt par la lecture. 
Le dernier de ses cours auquel j'assistai pour le plaisir et non pour les crédits portait sur la poésie française de 1900 à 1920 - autour de la figure d'Apollinaire. J'en ai le plan détaillé devant moi et je constate que le professeur relevait avec érudition le défi de "faire un petit sort" à tous les "ismes" qui se sont multipliés dans la période. Il s'agissait évidemment d'un cours d'histoire littéraire où Jacques Brault, toutefois, tirait parti des chronologies, ramenait à bon droit le biographique, préconisait les anthologies d'auteur tout en usant d'outils conceptuels qu'il faut assouplir, tels que les notions de siècle et de génération. Effectivement, il ne se trompait pas en soulignant au passage le matériel autobiographique dans les poèmes d'Apollinaire, lui qui a écrit tout près de son existence et de son époque et dont certains cycles de l'œuvre coïncident avec les cycles amoureux de sa propre vie. Dans tous les cas, ce qu'il y a de particulier à Jacques Brault, lorsqu'il fait de l'histoire littéraire, c'est qu'il ne réduit pas: le concept de mouvement ou le critère temporel de vingt ans qui décide d'une génération sont pour lui plus arbitraires qu'infaillibles, étant donné que les générations intellectuelles sont définies surtout par les affinités conscientes ou non et par une certaine pratique commune dans les auvres. Ainsi, de l'intérieur, Catherine Pozzi pouvait être plus près de Louise Labé que de bien d'autres...

Pour nous amuser, il avait parfois de ces télescopages, pour ainsi dire, comme dans cette notice biographique: Marie Louise Antoinette de Heredia, Gérard d'Houville de son nom de plume, fille du poète parnassien mariée à Henri de Régnier et maîtresse de Pierre Louïs qui était le mari de sa sour. On était presque dans la dégénérescence des unions consanguines. Impayable aussi cette petite charge dont la théorie du texte faisait les frais et où il nous mimait le "x»-du mot "texte» justement - esseulé, isolé au bout de la ligne. Il protestait contre le langage qui s'auto-suffit, qui ne réfère plus, n'a plus de sujet. À ce propos, il avait une boutade - qui pourrait représenter un historique de la question posée par les psychanalystes: Dans le texte, quel est l'inconscient que l'on psychanalyse? - On parle, ca parle, parle; et il attendait la suite, la consonne occlusive seule: /p/. Mais Jacques Brault visait avant tout, à travers ces quelques moqueries, la contradiction suivante: Le langage littéraire se veut intransitif, pure nomination et émanation, bien qu'en même temps, comme tout langage, il se veut transition et que le souci de communiquer l'emporte; il faut donc que la poésie reste langage. garde sa sociabilité. Sans quoi il y a risque d'échouer sur l'écueil de la poésie pour les poètes ou pour les initiés, de faire usage d'un charabia pour les "happy few».

C'est un peu pour toutes ces raisons qu'il ne cachait pas sa sympathie pour les fantaisistes Carco et Toulet - surtout Toulet - marqués au coin d'un vouloir écrire clair et net, transparent. Leurs poèmes courts se donnent pour peu de chose et s'arrangent d'un laisser-aller d'apparence misant sur quelques éléments, sur un ou deux échos. Il en allait de même de Villon dans un autre cours: nous glissions vers l'irréductible, à la recherche du lieu et de la formule, du trait de tous les traits, du noyau matriciel auquel, au-delà des techniques d'écriture, nous avons difficilement accès. Jacques Brault ne ramenait pas un auteur à un mouvement ou à une époque, mais il allait à sa 
singularité d'être écrivant avec laquelle se rencontrait sa singularité d'être lisant investi d'une parole, d'une culture, d'un sens de la philosophie et de l'histoire tout à fait hors du commun.

Dans son cours sur l'intimisme, il nous apprenait la lecture et nous entretenait de l'écriture:

Pour écrire, il suffit d'une grammaire et de n'importe quoi. disait-il; le problème, c'est de les joindre. Il n'y a pas que le travail ou le don, puisque cela, qui est acquis, il faut le perdre / il aurait pu nous citer Jean de la Croix: "Pour aller où tu ne sais pas, va par où tu ne sais pas (Chemin faisant, p. 143)." / ; il faut surtout une présence absolue et radicale à ce trésor intime qu'on voudrait garder et qu'il importe néanmoins de placer dans un jeu de médiations et de processus. Il faut chercher ce qui relie l'individualité la plus individuelle et la communauté la plus profonde.

Ailleurs, traitant de l'œuvre de Miron, il réaffirmait son adhésion aux mots proches de la vie quotidienne entendue comme /.../ l'affirmation écrite-vécue que nous sommes faits d'une même étoffe (à l'instar de nos songes); et pour un qui meurt nous mourons tous, et pour l'autre qui renait à sa persistance tous nous réintégrons la demeure d'une seule existence. L'homme quotidien, c'est chacun des hommes en l'homme et inversement /.../ réciprocité du pluriel et du singulier (Chemin faisant, p. 24-25). Ou encore: $A$ l'exemple de Gabrielle Roy, en n'évacuant pas la banalité du quotidien, il faut traverser les apparences et les apporter avec soi au fond, favoriser l'expansion par le creux de l'intimité, la "communicabilité" dans l'en dessous. L'écriture est un mouvement de repli qui s'ouvre par le fond. Avant tout, bien sûr qu'il faut avoir quelque chose à dire, quelque chose qui, si possible, parte d'un centre affectif et irradie dans toute l'ouvre - comme l'enfance matricielle de Gabrielle Roy - permettant ainsi d'éviter le piège rationnel du dire sur le dire - gauchissement de Mallarmé.

Et une fois admise cette nécessité de se perdre en se propageant, il faut encore s'enfermer et n'attendre rien: il faut sécréter son propre courage. Mais cet affrontement de la solitude écrivante fait peur aux écrivains. On risque de ne plus jamais remonter à la surface, et de devenir pour toujours inutilisable. Pis encore: on risque de se laisser prendre au texte (mais c'est pourtant le risque du lecteur) et de ne plus être bon à rien qu'à écrire (Chemin faisant, p. 79). Il a toujours insisté sur la solitude. Je devinais qu'il devait se recueillir souvent, même en présence d'un groupe, s'absenter à la faveur de quẹques "moments fragiles» qui sont des haïku par l'intention. Son inspiration. je la pressentais distribuée dans son quotidien; à sa table d'écriture, me disais-je, il travaille...

Dans «tout dire», tout n'égale pas l'addition de mille manies dont on rougirait en public. Tout, c'est plutôt un risque... Il songeait à Rimbaud, moi, je pensais à Mauriac qui, dans son entendement, risquait son salut, à Jouhandeau qui encourut l'ostracisme, à Émily Brontë... Oui, nous dialo- 
guions - en silence. Et il ajouta: Quand on a tout dit, il se peut qu'on n'ait plus rien à dire...

Il nous enseignait comment lire. Dans un premier temps, cela prenait quasiment l'aspect d'un rituel. D'abord, nous confiait-il, ne s'occuper que de la langue et non des idées, c'est là une manière de reconnaître superficiellement la profondeur - s'il y en a une. Puis, se contraindre soi-même par une mise au foy'er, s'abstraire du bruit, de la rumeur. Je le voyais suivre le texte - de Cabanis cette fois-là - en jouer, tout en devinant qu'il avait dû le laisser jouer aussi sur lui-même, le poursuivre. Il souhaitait que l'on se dise sa lecture dans des silences articulés, dans une semi-diction silencieuse, dans une sorte de demi-soliloque ou de rumination, puisque ce sont de telles répercussions qui ont la chance d'inquiéter nos concepts. Ce qu'il appelait aussi garder le silence, le garder avec des mots-murmures; des mots émus de l'innommahle (Trois fois passera, p. 78). Il voulait qu'on aille à l'intimité du texte à travers sa propre intimité d'être lisant, car ce dont il est toujours question. c'est du mal d'être au monde, et voilà la question quarticule la littérature. Si j'ai bien compris, à la fin, nous n'écrivons que pour avouer l'inavouable: nous sommes faits pour le bonheur, nous ne sommes faits que pour ca, et nous ne sommes pas heureux, pas vraiment - pas encore (Trois fois passera, p. 53).

La littérature ne s'apprend pas, elle se cultive. Tout ce qui. à la rigueur. s'apprend, c'est la lecture plus spécifiquement littéraire de l'œuvre littéraire. Ils'agit de la lecture qui aiguise toute la perception et l'intellectualité - et qui peut devenir lecture critique. Là-dessus, justement, ce très beau texte: Le cceur de la critique est fait d'abord de sentiment. N'est-il que sentiment? Le sentir, à l'opposé du savoir, n'opère bien que dans la proximité et encore plus dans l'homogénéité. Un mot de Sénèque résume les effets de la sympathie: toute amitié trouve l'égalité ou la crée. Quand la critique se veut "créatrice", elle donne à entendre qu'il ne lui suffit pas de comprendre et de juger; elle veut plus et mieux: être heureuse du même bonheur que locuvre, et d'un bonheur de langage (Chemin faisant, p. 57). Jacques Brault s'est toujours avoué partisan d'une lecture totale, libérée, risquée, située dans un inconfort par la variation de ses points de vue. Une lecture de recréation. Oui, ici, lire et écrire vont de pair, sont inséparables. En présence d'un monument, le lecteur cherche à en édifier un autre dans et par sa parole.

Mais cela se fait, il me semble, au prix d'un consentement: il faut accepter qu'au bout du texte de critique comme au bout du texte de création appelons-les ainsi pour simplifier - il y ait deux porte-plume ou, pourrait-on dire, des porte-lampe: deux solitudes qui se font signe. Il faut accepter que la critique s'objective un peu à la lumière d'une herméneutique, c'est-à-dire dans un mouvement de va-et-vient entre l'intuition du lecteur et le texte qui la fonde, va-et-vient entre le texte fondé et le fondateur. Alors, et alors seulement, notre lecture pourra recouvrer la dimension humaine de l'écriture de même qu'elle pourra récupérer l'expérience, la connaissance et le savoir intimes. Nous aurons découvert, dans l'intensité affective, plusieurs relais de la raison aptes à l'enrichir et nous aurons éprouvé davantage que l'on est toujours plus intelligent ou lucide à parler de ce que l'on aime. 
Jacques Brault n'aimerait pas que je le place au-dessus de son ceuvre: à la vérité. l'un et l'autre me requièrent. Je ne connaissais pas le professeur que j'enviais déjà les titres heureux de l'écrivain: la Poésie ce matin. l'En dessous l'admirable. Poèmes des quatre côtés... Maintenant. ils sont indissociables. Quand je lis l'un. j’entends l'autre: je les perçois ensemble. réunis par le ton - comment dire? - oui. le ton de la "littérature confidentielle". Dans des lieux privilégiés de son auvre. le temps d'une phrase comme celle qui vient. sa voix de chair double la voix de papier et elles effacent discrètement la mienne: IIy en a qui se consacrent aux grandes choses - et ie les admire: ily. en a qui s'accordent avec les petites choses - et je les aime (Trois fois passera. p. 74). Je ne croirais aux entretiens que pour cette grâce. ce "fading" qui opère. par la suite, dans mes lectures. On lit bien différemment les écrivains que l'on connaît puisqu'en plus du texte, on entend ce qu'ils pensent.

Ce qui maa toujours fasciné chez Jacques Brault. c'est qu'il croit en la belle parole - pas celle qui est voulue, mais l'autre qui advient. Il donne accès à cette parole au plus grand nombre en renouvelant. entre autres choses. nos lieux communs, en nous les rendant acceptables. Resimboliser ce qui ne symbolise presque plus, c'est un peu le travail de la poésie: briser le sens commun pour remonter à un sens plus près de lorigine. Pour c'e faire. le poète sinstitue peut-être davantage sı̣ntacticien que lexicologue. Cette faculté langagière n'est-elle pas l'offrande la plus souhaitable qu'un poète puisse rapporter à sa collectivité? Il extrait, du quotidien d'ici, le merveilleux pour ici, pour ailleurs.

Mais pour ma part. Jacques Brault m’a surtout donné une chose et j’ai voulu la lui rendre en ce pauvre hommage: une caution d"écriture. Les essais et les cours de Jacques Brault répondent d'une attitude critique qui ne se réalise pleinement que dans le langage.

J'ai ouvert à nouveau ses livres et c'est toujours le même charme qui se manifeste. le même que dans cette leçon élargie dans laquelle j’ai voulu le montrer près des choses qui nous entourent: on sent quelqu'un. Il n'y a qu'à relire la page soixante et onze de Chemin faisant pour achever de s'en convaincre. Oui! écrivez encore, Monsieur, car quand je pense à vous je me redis souvent cette phrase de Jankélévitch:

Pour une fois il s'avère, ô miracle! que quelqu'un a quelque chose à dire.

(Quelque part dans l'inachevé. p. 41)

1. Sauf indication contraire, toutes les citations de cet article sont extraites de mes notes de cours. 


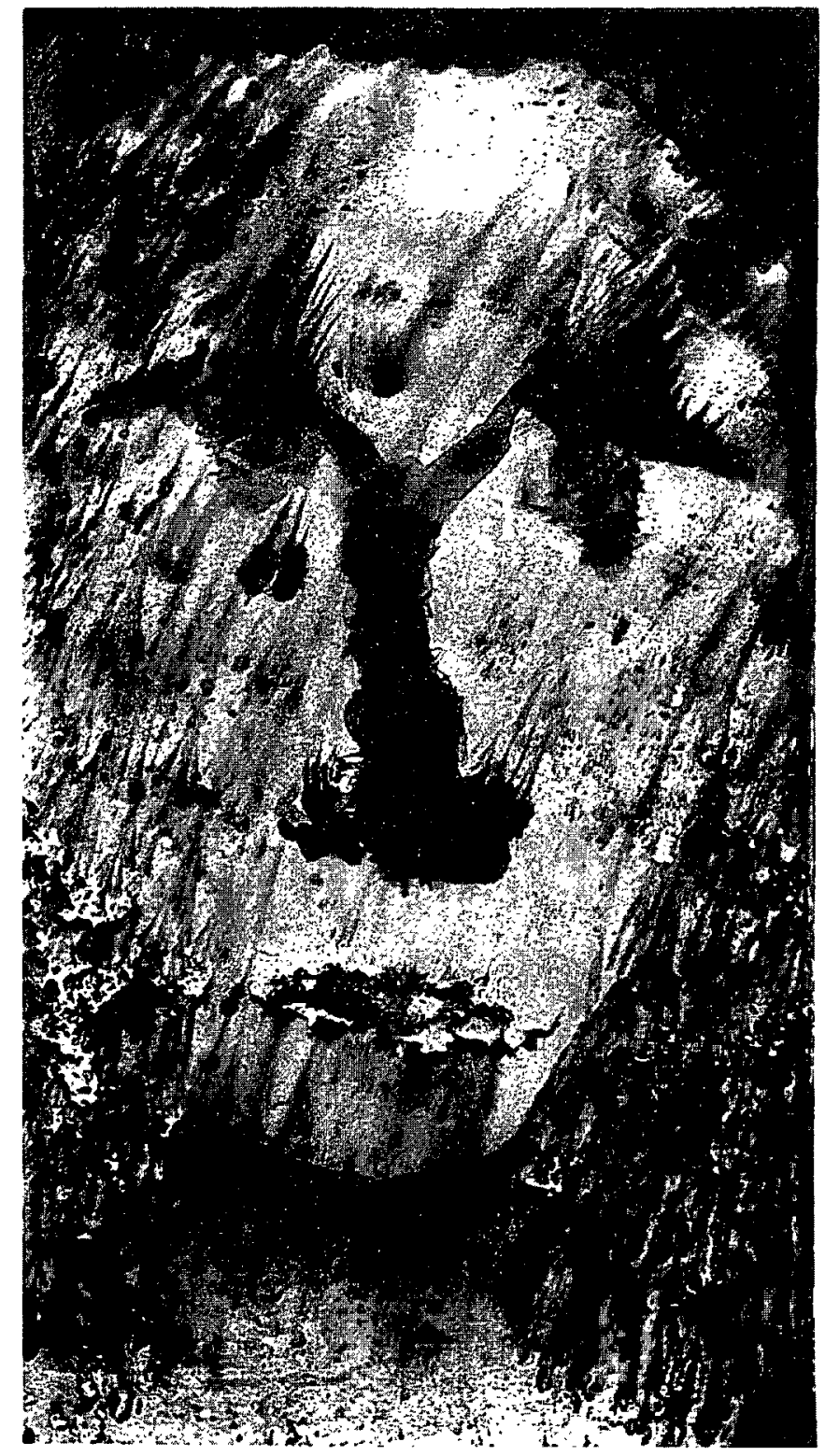

